\title{
Anseios dos familiares relacionado a implantação de gastrostomia em pacientes com incapacidade cognitiva
}

\section{Relatives' anxieties related to gastrostomy implantation in cognitively impaired patients}

DOI: $10.54022 /$ shsv2n3-014

\section{Douglas Geraldo Ferreira}

Enfermeiro graduado pela Faculdade Ciências da Vida-FCV, Sete Lagoas/MG;

Especialista em Urgência Emergência e Trauma pela PUC Minas;

Especializando em Estomaterapia pela UNIREDENTOR/ IESPE - Juiz de Fora/MG.

\section{Clebio Dean Martins}

Enfermeiro graduado pela Faculdade Ciências da Vida- FCV, Sete Lagoas/MG; Especialista em Urgência Emergência e Trauma pela PUC Minas; Mestre em Saúde e Educação pela UNAERP- Ribeirão Preto/ SP; Docente do curso de Enfermagem da Faculdade Ciências da Vida. Orientador da pesquisa.

E-mail: clebiodean@oi.com.br

\section{Denise Lembi Ferreira}

Graduada em Medicina UFMG, Pós Graduada em Nefrologia HCFMRP-USP, Especialista em Nefrologia, Pós Graduada em Promoção da Saúde -Fundação Unimed, MBA Executivo Internacional em saúde FGV, Gestora Viver Bem /Unimed Sete Lagoas/MG.

E-mail:delembi@gmail.com

\section{RESUMO}

Contextualização do tema: A implantação da Gastrostomia requer uma indicação médica ou de um profissional nutricionista. Desde que o paciente apresente condições clinicas e funcionais para tal, pois é um ato invasivo, seja pelo seguimento percutâneo ou cirúrgico (NAVES, 2014). Objetivo: Compreender os fatores que interferem na implantação da Gastrostomia de pacientes assistidos por um programa de atendimento domiciliar com incapacidade cognitiva à partir dos relatos de familiares. Materiais e Métodos: Estudo qualitativo, descritivo, analítico e exploratório, realizado em uma operadora de plano de saúde de um município do interior de Minas Gerais. Sendo que as 07 entrevistas foram 
aplicadas aos familiares de pacientes com incapacidade cognitiva, com diagnóstico de demência do tipo Alzheimer, com Extrato Clínico Funcional 09 e 10, assistidos pela equipe de Assistência Domiciliar do Viver Bem da Unimed Sete Lagoas/MG, denominada Gerenciamento de Casos Especiais. Resultados: A análise dos dados seguiu a proposta de análise temática de conteúdo de Bardin (2016), onde emergiram duas categorias: "Gastrostomia: Implantação favorável ou desfavorável em pacientes com incapacidade cognitiva sob o ponto de vista dos familiares" e "Prolongamento de sofrimento e terminalidade da vida de forma digna: percepção dos familiares em relação à Gastrostomia". Considerações finais: Esta pesquisa, demonstrou os desfechos relacionados a implantação ou não do dispositivo Gastrostomia em pacientes portadores de demência e incapacidade cognitiva, no qual apresentam, por conseguinte a perda da autonomia para decidir pelo procedimento médico. Diante disto, foram observados vários relatos de sentimentos como anseios, preocupações, dificuldades na tomada de decisão, medo, necessidade, prolongamento e confortabilidade. Concluiu se que, o objetivo de conhecer intrinsecamente os familiares de pacientes com incapacidade cognitiva e sua relação com a implantação do dispositivo Gastrostomia foi alcançado com excelência, visto que os questionamentos nortearam uma reflexão e, os familiares expressaram suas vontades, culturas e percepções no processo do cuidar.

\section{Palavras-chave: Estomaterapia; Gastrostomia; Familiares; Anseios}

\section{ABSTRACT}

Contextualization of the theme: Gastrostomy implantation requires medical indication or from a nutritionist. As long as the patient has clinical and functional conditions for this, as it is an invasive act, either by percutaneous or surgical followup (NAVES, 2014). Objective: Understand the factors that interfere with the implementation of Gastrostomy of patients assisted by a home care program with cognitive disability from the reports of family members. Materials and Methods: Qualitative, descriptive, analytical and exploratory study, conducted in a health insurance company in a city in the interior of Minas Gerais. The 7 interviews were applied to the relatives of patients with cognitive disability, diagnosed with Alzheimer's dementia, with Estrato Cliníco Funcional 09 e 10, assisted by by the Viver Bem at Unimed Sete Lagoas / MG, called Gerenciamento de Casos Especias Results: Data analysis followed Bardin's (2016) proposal for thematic content anaysis, where two categories emerged: "Gastrostomy: Favorable or unfavorable implantation in patients with cognitive disability from the family point of view" and "Prolonging suffering and terminality" of life in a dignified manner: family members' perception of Gastrostomy "Final considerations: This research demonstrated the outcomes related to the implantation or not of the Gastrostomy device in patients with dementia and cognitive disability, in which they present the loss of autonomy to decide on the medical procedure. Given this, several reports of feelings such as anxieties, concerns, difficulties in decision making, fear, need, prolongation and comfort were observed. It was concluded that the objective of intrinsically knowing the relatives of patients with cognitive disability and their relationship with the implantation of the Gastrostomy device was achieved with excellence, since the questions guided a reflection and the relatives expressed their wishes, cultures and perceptions in the process Caring. 
Keywords: Stomatherapy; Gastrostomy; Relatives; Yearnings

\section{INTRODUÇ̃̃O}

Comumente um indivíduo nas suas funções fisiológicas e com necessidades nutricionais para sobrevivência, utiliza a via oral convencional para introduzir sua alimentação em geral, salvo em condições especificas que o restringe, e neste contexto se faz necessária a confecção de uma via alternativa como a Gastrostomia, sendo esta, por ato cirúrgico ou endoscópico, onde se é criado uma luz comunicando o meio externo até o sítio interno do estômago através de uma sonda (SANTOS, 2011; MARTINI, 2013).

A implantação da Gastrostomia requer uma indicação médica ou de um profissional nutricionista. Desde que o paciente apresente condições clinicas e funcionais para tal, pois é um ato invasivo, seja pelo seguimento percutâneo ou cirúrgico. É importante ressaltar que é indicada quando o indivíduo necessitar de suprir moléculas de nutrientes em um período maior que seis semanas e que após a sua implantação o paciente apresente condições favoráveis para estabilização e manutenção da sua saúde nutricional (NAVES, 2014).

Os familiares de pacientes com incapacidade de tomar decisões próprias, por processo demencial e cognitiva, se deparam frequentemente com conflitos relacionados ao seguimento da Estomaterapia, dentre eles a Gastrostomia, sendo que, está implantação deve ser necessária e favorável à dignidade humana, ou seja, não prolongar o sofrimento do paciente. Então, é crucial o acompanhamento por uma equipe multidisciplinar indiferentemente da formação, juntamente com os familiares envolvidos na coordenação do cuidado e ainda, que tenham uma estreita comunicação com o paciente, quando houver esta possibilidade do diálogo (WEBER et., al 2017).

Para o paciente assistido por uma equipe multidisciplinar, a discussão é feita inicialmente em âmbito domiciliar e logo após a definição da sua confecção, o mesmo é encaminhado para o hospital, contudo, no momento da alta, onde os familiares estão repletos de dúvidas e incertezas de como cuidar e manipular o estoma gástrico, percebe-se que estas informações, as vezes são esclarecidas de forma apressada e sem clareza, por vezes pelo profissional generalista e não pelo Estomaterapeuta. Portanto, devemos assegurar que os familiares possam dar seguimento nos cuidados no domicílio, porém e uma conduta que está em 
adaptação e carece de averiguação sistemática de todos profissionais envolvidos no cuidado (WEBER et., al 2017).

A mudança do modelo assistencial favoreceu alterações no acolhimento e atendimento dos pacientes com várias necessidades e, uma delas foram os cuidados domiciliares, visto que é oferecido uma assistência pelos familiares com apoio de uma equipe multidisciplinar (MUNIZ et al., 2014).

O destaque mais importante na assistência domiciliar é proporcionar ao indivíduo e sua família um apoio próximo, acolhedor e esclarecedor, enfatizando e promovendo um vínculo de confiança para uma melhor recuperação, reabilitação e tratamento. Necessário destacar que esta permanência na residência traz benefícios como diminuição da ansiedade de uma hospitalização, com consequente melhoria na qualidade de vida ou até mesmo, melhoria na qualidade de morte em alguns casos. Segundo Silva et. al (2015) o modelo que assiste o paciente no domicilio na Europa cresceu, e os números apontam que o domicilio é o principal sitio de terminalidade.

A equipe do Viver Bem da Unimed Sete Lagoas/MG oferece uma assistência multiprofissional no sentido de orientar, acolher, acompanhar e monitorar os pacientes com necessidade de implantação da Gastrostomia, além de esclarecer e apoiar a tomada de decisão dos familiares, bem como assistir os pacientes após a sua implantação (ESCOPO VIVER BEM DA UNIMED SETE LAGOAS, 2019).

Assim, a presente pesquisa parte da questão norteadora: O que os familiares de pacientes com incapacidade cognitiva, pensam a respeito da implantação da Gastrostomia?

Partindo dos seguintes pressupostos: (i) Os familiares percebem a implantação da Gastrostomia como um recurso fundamental na manutenção da vida do paciente, independentemente da condição clínica do paciente; (ii) A implantação da Gastrostomia em dias atuais é um procedimento importante e necessário, porém deve-se ter indicação de um profissional médico, nutricionista, dentre outros, além dos esclarecimentos dos familiares, paciente e sobretudo da aceitação e entendimento conjunto. (iii) Os familiares não aceitam a implantação da Gastrostomia, uma vez que é um procedimento invasivo e oferece riscos, sendo que a não implantação do dispositivo possibilita uma terminalidade da vida 
de forma digna.

Tem por objetivo geral compreender os fatores que interferem na implantação da Gastrostomia de pacientes assistidos por um programa de atendimento domiciliar com incapacidade cognitiva à partir dos relatos de familiares. E por objetivos específicos Descrever a Escala Analógica da Fragilidade Clínico-Funcional utilizada com pacientes no Programa de Assistência Domiciliar e observar o papel da família na tomada de decisão relacionado à Gastrostomia em pacientes com incapacidade cognitiva.

A relevância deste estudo constitui-se pelos vários sentimentos que perpetuem este contexto da indicação de Gastrostomia, dentre os quais, podemos citar o ato de decidir e responder pelo outro por causa da incapacidade cognitiva, outras questões socioculturais e também a carência de informações dos familiares e cuidadores no que tange o cuidado com o estoma gástrico. Com isso observase uma demanda permanente para a orientação e continuidade deste cuidado no domicilio, que possa contemplar as necessidades do paciente de forma lucida, integralizando toda a família e cuidadores. "Independentemente de ser temporária ou definitiva, a realização desse procedimento acarreta uma série de mudanças na vida do paciente e requer um cuidado especializado de enfermagem" (GOMES E MARTINS, 2016). Contudo, nesta oportunidade 0 enfermeiro estomaterapeuta, deve promover práticas educativas de modo que ofereça escuta individualizada e personalizada aos familiares e pacientes, além de envolverem e tranquilizarem para a nova situação de vida.

\section{REFERENCIAL TEÓRICO}

\subsection{IMPLANTAÇÃO DA GASTROSTOMIA: INDICAÇÕES E CONDIÇÕES PERANTE O DECLÍNIO FUNCIONAL}

A confecção da Gastrostomia pode ser realizada por meio endoscópico, radiológica, laparoscópico ou por laparotomia, no qual irá depender da indicação do profissional médico que acompanha o caso, pois são várias as indicações para esta via alternativa. É importante ressaltar que pode também ser utilizada como via de descompressão gástrica para pacientes com neoplasias abdominais que como consequência podem ocluir o trânsito intestinal (RODRIGUES et al., 2018).

A equipe multidisciplinar tem um papel fundamental no acompanhamento 
destes pacientes submetidos a implantação da Gastrostomia, bem como dos seus familiares, pois será comum o aparecimento de condições que trará duvidas e ansiedade no manejo deste estoma e nesta direção, cabe a equipe multidisciplinar planejar o atendimento a estas pessoas, levando em consideração seus conceitos biopsicossociais, culturais e espirituais. Diante disto, o cuidado se torna essencial para antecipar as complicações e no surgimento, tratar o mais precocemente possível (RODRIGUES et al., 2018).

\subsection{ATENDIMENTO DOMICILIAR VIVER BEM DA UNIMED SETE}

\section{LAGOAS/MG}

O Programa de Atendimento Domiciliar da Unimed Sete Lagoa/MG, denominado como Gerenciamento de Casos Especiais é pautado na assistência domiciliar programada, ou seja, são oferecidos atendimentos por uma equipe multidisciplinar de segunda à sexta-feira, em horário comercial, exceto sábado, domingo ou feriado. É um ramo da atenção à saúde que envolve ações de promoção da saúde, prevenção e tratamento de doenças no âmbito domiciliar, com foco no atendimento ambulatorial dos beneficiários que apresentem limitação funcional (ESCOPO GERENCIANTO DE CASOS ESPECIAIS VIVER BEM DA UNIMED SETE LAGOAS/MG, 2019).

Para monitoramento e acompanhamento dos pacientes assistidos pelo atendimento domiciliar é aplicada uma escala que compõe a avaliação multidimensional do idoso, sendo denominada Escala Analógica da Fragilidade Clínico-Funcional (Anexo 1), no qual é possível classificar os idosos em 10 (dez) estratos, sendo que, quanto maior o estrato, mais elevados a fragilização e o declínio funcional. Os extratos 1,2 e 3 são classificados como Robustos; 4 e 5 Em risco de Fragilização (CARMO, 2014).

Nos estratos de 1 a 3, são categorizados os Idosos Robustos, ou seja, aqueles que possuem capacidade de conduzir as Atividades de Vida Diárias (AVD) de modo independente e autônomo; já os estratos 4 e 5, são compostos pelos Idosos em Risco de Fragilização, que administram todas as AVD também com autonomia e independência, entretanto, encontram-se na transição da senescência (envelhecimento fisiológico) e senilidade (envelhecimento patológico), ocasionando algumas limitações funcionais resultantes desse 
processo, podendo ainda apresentar alguma patologia crônica; por fim, 6 e 7 Frágil de Baixa Complexidade, 8 de Alta Complexidade e 9 e 10 em Fase Final de Vida, sendo estes incapazes de administrar sua vida (dependentes de cuidados, sobretudo de alimentarem-se sozinhos), com declínio funcional presente (CARMO, 2014).

\section{MÉTODOS}

O presente trabalho foi um estudo descritivo, analítico, exploratório, qualitativo, no qual as entrevistas foram agendadas previamente entre o período de maio a junho de 2019, através de um questionário semiestruturado elaborado pelos próprios autores, sendo aplicado para os familiares dos pacientes assistidos no domicílio pelo programa de gerenciamento de casos especiais do Viver Bem da Unimed Sete Lagoas/MG. Os participantes foram escolhidos pelo critério de conveniência e a coleta de dados se deu por entrevista com um roteiro semiestruturado e a interrupção das entrevistas se deu pelo critério de saturação

Foram avaliados 7 familiares de pacientes que foram classificados na escala de Extrato Clínico Funcional 09 e 10 e que tem o diagnóstico de demência do tipo Alzheimer, sendo assistidos no domicílio pelo Viver Bem da Unimed Sete Lagoas/MG, no qual o responsável legal apresentasse idade $\geq 18$ anos e que aceitaram participar da pesquisa e assinaram o termo de consentimento livre e esclarecido (TCLE).

Já os critérios de exclusão foram os familiares de pacientes com Extrato Clínico Funcional $\leq$ 08, que não tem o diagnóstico de demência do tipo Alzheimer e o responsável legal; com a idade $\leq 18$ anos e que não aceitaram participar da pesquisa com assinatura do Termo de Consentimento Livre e Esclarecido (TCLE). Destaca-se que essa pesquisa seguiu os parâmetros éticos segundo a Resolução 466/2012 e todos os participantes assinaram o TCLE em duas vias, após os familiares serem orientados sobre os objetivos e motivo da pesquisa. Posteriormente a análise dos dados seguiu a proposta de análise temática de conteúdo de Bardin (2016), onde emergiram duas categorias: "Gastrostomia: Implantação favorável ou desfavorável em pacientes com incapacidade cognitiva sob o ponto de vista dos familiares" e

"Prolongamento de sofrimento e terminalidade da vida de forma digna: percepção 
dos familiares em relação à Gastrostomia".

Para manter o anonimato dos participantes, os discursos dos familiares foram identificados de F1 a F7.

Trabalho submetido à Plataforma Brasil, aprovado, com CAAE 40698019.9.0000.8164, Versão 1.

\section{RESULTADOS E DISCUSSÃO}

Os entrevistados para a pesquisa foram 7 familiares de pacientes assistidos pelo Atendimento Domiciliar do Viver Bem da Unimed Sete Lagoas/MG, sendo 05 mulheres e 02 homens, com idade entre 18 e 100 anos. As entrevistas foram elaboradas com seis questões discursivas, relacionadas a temática no qual surgiram três categorias, conforme descrição no quadro I.

\section{Quadro I: Categorias da Análise de Conteúdo}

I - "Gastrostomia: Implantação favorável ou desfavorável em pacientes com incapacidade cognitiva sob o ponto de vista dos familiares"

II - "Prolongamento de sofrimento e terminalidade da vida de forma digna: percepção dos familiares em relação à Gastrostomia”.

Fonte: Dados da pesquisa, 2019.

\subsection{Gastrostomia: implantação favorável ou desfavorável em} pacientes com incapacidade cognitiva sob o ponto de vista dos familiares.

A atenção domiciliar requer uma assistência próxima e contínua, com um olhar diferenciado e atento para possíveis complicações no que tange ao paciente portador de Gastrostomia e, nesta ótica que devemos integrar os familiares de forma que eles próprios possam identificar alterações e dificuldades pontuais no processo de alimentação diária, no sentido de estreitar a relação, no acompanhamento e auxílio relacionado às dúvidas e anseios dos familiares nesta redescoberta de uma nova forma de cuidar.

"A Gastrostomia surge então como um procedimento que visa fornecer o aporte nutricional por um acesso direto ao estômago, através da introdução cirúrgica ou endoscópica de uma sonda" (SANTOS, 2011; MARTINI, 2013).

Tyng (2017) ressalta que o objetivo é criar um acesso para introduzir nutrientes em pacientes que não permitem mais a via oral para alimentação e que nesta situação manifestará condições de riscos para deficiência nutricional. 
A Gastrostomia é um procedimento que requer uma hospitalização pela sua complexidade, porém após a sua implantação, o paciente retorna para o seu domicílio. A internação para a implantação deste dispositivo se torna necessária, mas muitas vezes por mais que o paciente apresente a necessidade, a sua consulta à nível ambulatorial é repleta de dúvidas, seja pelo paciente ou pelos familiares, e até mesmo quando este não puder tomar a sua própria decisão devido sua dificuldade cognitiva. As falas abaixo demostram o entendimento dos familiares em relação à Gastrostomia. As falas abaixo demostram este entendimento dos familiares.

\begin{abstract}
E um processo, um procedimento que você faz, quando a pessoa tem dificuldade de deglutição ou outros comprometimentos para poder alimentar via sonda ligada no aparelho gástrico. (F6)
\end{abstract}

No caso é a sonda que é colocada e através dela o paciente recebe o alimento. (F2)

Gastrostomia seria alimentação através de sonda, para a pessoa que não consegue ingerir o alimento de forma natural, de forma correta, então seria alimentar através de sonda. (F3)

Comumente, o que vemos e escutamos é que a indicação principal para confeccionar um estoma gástrico é para alimentar-se, ou seja, quando contraindicada o ato por via oral, seja ele por deficiência na deglutição ou por outros motivos. O entendimento de como é realizado o procedimento é importante para que a família possa optar com leveza e lucidez o melhor caminho juntamente com a equipe e o médico de referência. Por outro lado, o que se percebe através da pergunta supracitada é que os cuidadores tem uma noção simples porém objetiva de como é realizado a implantação de uma Gastrostomia.

No caso destes pacientes com déficit cognitivo devido a um processo demencial já instalado, particularmente repercute em diferentes modos de abordagem e entendimento no sitio familiar, pois o ensejo da patalogia ocasiona grandes impactos no seio familiar e na sociedade em geral, ainda mais quando 0 indivíduo perde sua autonomia e capacidade de decidir (TALMELLI, 2010).

Segundo Cruz; Ângelo; Gamboa (2012) é necessário entender que mesmo que a via alternativa possa trazer suas vantagens, principalmente uma melhor qualidade de vida, fica ainda uma incerteza dos familiares e cuidadores em relação ao risco e benefício para a implantação da Gastrostomia, portanto, é neste contexto repleto de sentimentos, que consequentemente dificulta a decisão. Pode 
se identificar tais circunstâncias pelos relatos descritos abaixo concernente ao entendimento dos familiares em relação aos pontos favoráveis e desfavoráveis e suas percepções na implantação da Gastrostomia.

Acho que o paciente perdendo nutrientes, alimentação é responsável pela vida, e de extrema necessidade, quando ele não der conta, para ter uma qualidade de vida melhor. (F1)

Eu vejo a necessidade da implantação, se não tiver outra alternativa quando precisar alimentar e não tiver outra forma menos agressiva ou mais fácil de poder ingerir alimentos e a única saída, sim e valido. (F7)

Na situação do meu pai eu não permitiria implantar, eu quero e ele já deixou por escrito que não quer nada artificial para prolongar a vida. (F4)

Se fosse indicado pelo médico e fosse necessário para prolongar a vida de minha mãe eu não hesitaria. (F5)

Segundo Soares (2016) no caso do ser humano, a alimentação vai além desse caráter de sobrevivência. Tornou-se um ritual cultural, de reunião, de partilha e de prazer. Nesta conjuntura, observa se que a maior parte dos familiares contemplam a necessidade de alimentar como fato primordial para manutenção da vida, trazendo consigo opiniões que levam a julgar que fariam a implantação da Gastrostomia com o parecer do profissional médico a qualquer custo.

Diante da situação de confortabilidade de decidir pelo próximo, qual condição será tomada e se essa condição é a melhor, são vários os fatores que integram este cenário. Nesta perspectiva, Derech e Neves (2018) ressaltaram que esta decisão em relação a via de alimentação pode ser compartilhada entre 0 profissional e o familiar para juntos chegarem em um melhor consenso na tomada de decisão. Neste propósito, os familiares dos pacientes que anuíram em participar da pesquisa foram questionados sobre se sentem confortável em tomar decisão para os pacientes assistidos, conforme relatos abaixo:

Confortável sim, não é fácil, acho que estas decisões são difíceis, mais como eu tenho uma compreensão, do quadro e das necessidades eu sinto confortável em decidir sobre os procedimentos. (F1)

Sozinha não. Somos 7 irmãos e a gente tem o mesmo amor, o mesmo carinho, a mesma vontade que ela viva muito, mais viva bem, sem sofrimento, sem dor, então e uma decisão para todos tomarem juntos e se for decidido que sim, acho que não tem outra saída, acho que se for o melhor para ela vai ser sim. (F2) 
para este tipo de procedimento, seria muito triste de saber que encaminharia para isto, mais também sabendo que seria a opção e que seria necessidade. Então eu iria sim concordar, não feliz lógico com este procedimento. (F3)

Me sinto muito confortável para não fazer, primeiro porque já conheço a posição dele e a minha bate coma dele, não precisamos prolongar a vida a qualquer custo, isto para nós é fundamental deixar que a vida encerre por si mesmo. (F6)

Não. Mas tomaria esta decisão após consultar meu irmão, meu marido, para maior segurança, visto que a decisão é difícil e de muita responsabilidade. (F5)

Foi neste dilema que os familiares levantaram sobre a complexidade que é tomar esta decisão, pois são vários os conflitos no ambiente familiar para chegar a um acordo e na sua maioria percebemos o conforto do representante familiar em decidir a via de alimentação do seu ente. Outra observação que merece destaque, é que os entrevistados enfatizaram que a decisão requer influência e participação de todos os envolvidos no cuidado direto ou indireto, para com isso chegar a um melhor desfecho. Em concordância com os relatos supracitados, Anselmo et. al (2013) acrescenta que a condição da instalação de uma Gastrostomia deve se levar em conta todas as particularidades de cada indivíduo e família, respeitando os conceitos técnicos e bióticos para cada caso, no intuito de diminuir os riscos e potencializar os benefícios.

\subsection{PROLONGAMENTO DO SOFRIMENTO E TERMINALIDADE DA} VIDA DE FORMA DÍGNA: PERCEPÇÃO DOS FAMILIARES EM RELAÇÃO À GASTROSTOMIA

A temática da terminalidade da vida busca muitos debates no comunidade cientifica, pois atualmente dilemas como dignidade e qualidade na finitude da vida, intervenções invasivas ou não invasivas ou situações como sessar a alimentação e hidratação pela via oral são amplamente discutidas entre os profissionais de saúde e familiares. Diante disto, nas vivências diárias as pessoas deparam-se frequentemente com desafios que proporcionará inúmeros sentimentos e emoções. 
uma doença potencialmente mortal. Previnem e aliviam o sofrimento, através da detecção precoce, avaliação e tratamento correto da dor e outros problemas sejam estes de ordem físico, psicossocial ou espiritual" (OMS, 2017).

Através das falas abaixo os familiares demostraram se a implantação da Gastrostomia é um recurso fundamental e se, a não implantação repercute em uma terminalidade da vida de forma digna.

\footnotetext{
É fundamental, a pessoa perde nutrientes no organismo como que ele vai ter a reposição, num estágio já as vezes avançado, de não conseguir mais alimentar,

então é super importante. (F2)
}

\begin{abstract}
Não acharia que seria um fim de vida digno não, porque seria obvio que ela estaria terminando seus dias, enfraquecendo por falta alimentação, por causa da alimentação adequada, por causa de um tratamento, digamos, eficaz, de um tratamento mais certo, que para mim seria a gastrostomia, talvez por ser o único meio, então eu acho que não seria uma forma digna de terminar os últimos dias não, seria muito triste, e eu acho triste quem convive com este tipo de situação, mais não seria digno não.(F6)
\end{abstract}

E difícil, mas eu penso sempre no que mãe vai sofrer menos, pois mais que seja doloroso para a gente pensar, que aquela decisão tomada vai terminar com a vida logo, eu acho que se for sem sofrimento e sem dor, eu acho que a gente tem que tomar a decisão de implantar a Gastrostomia. (F3)

Gastrostomia com certeza seria a opção pelo o que eu saiba para manter a vida da pessoa, claro que pode ser uma situação que está caminhando para o fim, porque indiferente da pessoa ingerir qualquer tipo de alimento, por via oral, via normal e eu acho que se e o recurso bom, ele é bem-vindo, não é o ideal ninguém quer para si ou para os outros, mais se é o recurso que existe para prolongar a vida da pessoa e bem vindo $\operatorname{sim}(\mathrm{F} 5)$

Para mim e pelo conceito dele que sempre foi assim, a não implantação vai permitir a ele uma terminalidade, um fim de forma mais digna. (F7)

E foi nesta circunstância que Neto (2010) ponderou a importância dos familiares saberem que naturalmente a pessoa perde a vontade de alimentar, e isto se dá pela progressão da doença e não pelo agravamento, sendo assim a introdução alimentar e a ingesta hídrica administrada de forma involuntária e imperiosa não vai fazer que melhore ou regride a doença, ao contrário, pode piorar o contexto e o que devemos priorizar é uma melhor qualidade de vida e bem-estar.

Cotrim (2012) reforça que tem sido bem aceita a introdução de sonda de gastrostomia principalmente como possibilidade de melhora no aporte nutricional do paciente, reduzindo a ansiedade do cuidador no momento da alimentação. 
Pinto (2012) reforça esta definição, descrevendo que em muitos casos, é relatado angústia dos familiares quando não são administrados alimentos ou líquidos ao paciente que encontra-se nos cuidados.

Em concordância com os relatos dos autores, é possível perceber através das falas dos familiares uma preocupação eminente no que se refere a perda da função de se alimentar, tendo em vista o ato de alimentar como um conforto e segurança. Em contrapartida, observa-se que houve também familiares cujas manifestações foram a favor da não implantação e, neste entendimento, mediante abster-se deste dispositivo, proporcionariam a terminalidade da vida de forma digna.

\section{CONSIDERAÇÕES FINAIS}

Esta pesquisa, demonstrou os desfechos relacionados a implantação ou não do dispositivo Gastrostomia em pacientes portadores de demência e incapacidade cognitiva, no qual apresentam, por conseguinte a perda da autonomia para decidir pelo procedimento médico. Diante disto, foram observados vários relatos de sentimentos como anseios, preocupações, dificuldades na tomada de decisão, medo, necessidade, prolongamento e confortabilidade.

Este assunto permite desdobrar futuras produções cientificas, principalmente na comunidade de pesquisa da Estomaterapia, uma vez que é um tema pertinente e a relevância dos achados é crucial para a construção do entendimento concernente á abordagem multiprofissional. Vale aprofundar nesta análise, que durante as entrevistas através de perguntas previamente estruturadas, o pesquisador estimulou os familiares a manifestarem suas vontades, desejos, incertezas e até mesmo choros e emoções, trazendo uma reflexão de como será a sua atitude no momento da implantação, mediado pela necessidade, será uma decisão unilateral ou compartilhada com os outros familiares? O médico, nutricionista, fonoaudióloga e demais da equipe multidisciplinar participarão do processo, com apoio e esclarecimentos? Neste cenário, é possível responder alguns pressupostos, dentre eles que os familiares percebem a implantação da Gastrostomia como um recurso fundamental na manutenção da vida do paciente, exceto uma familia, no qual não permite que o dispositivo seja implantado no seu familiar que se encontra em cuidados devido a 
piora geral da sua condição clínica, sendo que assim, a não implantação proporcionaria uma finitude de forma digna, menos invasiva e neste momento, desnecessária à manutenção da vida. Outro pressuposto alcançado, relaciona-se com a indicação médica ou nutricional, mas é perceptível através das falas dos entrevistados, que a implantação requer um entendimento conjunto e aceitação dos familiares, bem como esclarecimentos necessários e importantes que permitam uma escolha adequada no sentido de proporcionar uma dignidade humana.

Concluiu se que, o objetivo de conhecer intrinsecamente os familiares de pacientes com incapacidade cognitiva e sua relação com a implantação do dispositivo Gastrostomia foi alcançado com excelência, visto que os questionamentos nortearam uma reflexão e, os familiares expressaram suas vontades, culturas e percepções no processo do cuidar.

Esse estudo se limita aos 07 familiares de pacientes assistidos pela Equipe multidisciplinar do Programa Viver Bem da Unimed Sete Lagoas/MG. Implica em difundir o tema como propostas de planejamento e ações de uma equipe multidisciplinar a partir da compreensão de pacientes com incapacidade cognitiva, ou seja, com dificuldades na tomada de decisão. Sugere-se que, para futuros trabalhos, sejam investigados a Gastrostomia em cuidados paliativos em diferentes faixas etárias e quais os benefícios na condição de saúde dos indivíduos expostos a esta intervenção. 


\section{REFERÊNCIAS}

ANSELMO, C.B.; JUNIOR, T.V.; LOPES, L.R.; NETO, J.S.C. ANDREOLLO, N.A. Gastrostomia cirúrgica: indicações atuais e complicações em pacientes de um hospital universitário. Rev. Col. Bras. Cir. [online]. 2013, vol.40, n.6, pp.458462. ISSN 0100-6991. http://dx.doi.org/10.1590/S0100-69912013000600007. Disponível em: http://www.scielo.br/pdf/rcbc/v40n6/07.pdf. Acesso em: 10 de julho de 2019.

CASSETTARI, A.J.; MORITZ, R. D. Pacientes críticos elegíveis para avaliação paliativista. Arq. Catarin Med. 2015 abr-jun; 44(2): 60-73. Disponível em: http://www.acm.org.br/acm/seer/index.php/arquivos/article/view/26. Acesso em: 04 de maio de 2019.

CARMO, J. A. D. Proposta de um índice de vulnerabilidade clínico-funcional para a atenção básica: um estudo comparativo com a avaliação multidimensional do idoso. 2014 . Disponível em: http://www.bibliotecadigital.ufmg.br/dspace/bitstream/handle/1843/BUBDA4YHWT/disserta o completa juliana alves do carmo.pdf?sequence $=1$ Acesso em 10 de Março de 2019.

CRUZ, A.C.; ANGELO, M. GAMBOA, S.G. A visão da família sobre a experiência de ter uma criança gastrostomizada. Rev. Enf. Ref. vol.serllI no.8 Coimbra dez. $2012 . \quad$ http://dx.doi.org/10.12707/RIII1216. Disponível em:

http://www.scielo.mec.pt/scielo.php?script=sci arttext\&pid=S087402832012000300015. Acesso em: 15 de maio de 2019.

CRUZ, M.N.; HAMDA, A.C. O impacto da doença de Alzheimer no cuidador. Psicol. estud. [online]. 2008, vol.13, n.2, pp.223-229. ISSN 14137372. http://dx.doi.org/10.1590/S1413-73722008000200004. Disponível em: http://www.scielo.br/scielo.php?pid=S1413-

$73722008000200004 \&$ script=sci_abstract\&tlng=pt . Acesso em: 24 de julho de 2019.

ESCOPO. Programa Gerenciamento de Casos Especiais. Viver Bem Unimed Sete Lagoas/MG, 2019.

GOMES, E.J.C. O enfermeiro no orientar e cuidar do paciente idoso com gastrostomia em domicílio. Trabalho de Conclusão de curso de Pós Graduação de Enfermagem em Dermatologia. FACULDADE AVM. Orientadora: Chennyfer Dobbins Paes da Rosa. RIO DE JANEIRO, 2016.

Disponível em: http://www.artigos.com/artigos/21733-o-enfermeiro-no-orientar-ecuidar-do-paciente-idoso-com-gastrostomia-em-domicillio. Acesso em: 03 de maio de 2019.

INDÍCE DE VULNERABILIDADE CLÍNICO FUNCIONAL -IVCF-20 (Versão do profissional de saúde). Disponível em: https://www.ivcf-20.com.br/. Acesso em: 01 
de julho de 2019.

LIDIANE, N.R.; SILVA, A.M.O.; XAVIER, M.S.; CHAVES, E.M.C. Complicações e cuidados relacionados ao uso do tubo de gastrostomia em pediatria. ESTIMA, Braz. J. Enterostomal Ther., São Paulo, v16, e1018, 2018. Disponível em:

https://www.revistaestima.com.br/index.php/estima/article/download/464/pdf_1 . Acesso em: 25 de julho de 2019.

MUNIZ, E. A.; FREITAS, C.A.S.L.; ALBUQUERQUE, I.M.A.N.; LINHARES, M.S.C. Assistência domiciliar ao idoso no contexto Da estratégia saúde da família: análise da Produção científica

S A N A R E, Sobral, V.13, n.2, p.86-91, jun./dez. - 2014. Disponível em: https://sanare.emnuvens.com.br/sanare/article/download/578/311. Acesso em: 04 de junho de 2019.

NASCIMENTO, N.G.; BORGES, E.L.; DONOSO, M.T.V. Assistência de enfermagem a pacientes gastrostomizados baseada em evidências. $R$. Enferm. Cent. O. Min. VOL.5, NO 3, 2015. Disponível em: http://www.seer.ufsj.edu.br/index.php/recom/article/view/743. Acesso em: 11 de julho de 2019.

PINTO, F. J. C. Alimentação em final de vida: a opinião dos enfermeiros. Tese de Mestrado apresentada para a obtenção do grau de Mestre em Cuidados Paliativos, sob a orientação do Professor Doutor José Carlos Amado Martins. 2o CURSO DE MESTRADO EM CUIDADOS PALIATIVOS FACULDADE DE MEDICINA DA UNIVERSIDADE DO PORTO PORTO, 2012. Disponível em: repositorio.chporto.pt/bitstream/10400.16/1362/1/Pinto-F.pdf. Acesso em: 24 de julho de 2019

RESOLUÇÃO № 466, DE 12 DE DEZEMBRO DE 2012. Aprova as diretrizes e normas regulamentadoras de pesquisas envolvendo seres humanos e revoga as Resoluções CNS nos. 196/96, 303/2000 e 404/2008. Disponível em: http://bvsms.saude.gov.br/bvs/saudelegis/cns/2013/res0466 1212 2012.html

Acesso em: 12 de junho de 2019.

RODRIGUES, L. N. Construção e validação de tecnologia educativa para cuidadores de crianças com gastrostomia. Dissertação apresentada ao curso de Mestrado Acadêmico Cuidados Clínicos em Enfermagem e Saúde, do Programa de Pós-Graduação Cuidados Clínicos em Enfermagem e Saúde, do Centro de Ciências da Saúde da Universidade Estadual do Ceará. Orientadora: Prof. ${ }^{a}$ Dr. ${ }^{a}$ Edna Maria Camelo Chaves. Fortaleza, Ceará, 2017.

RUIZ, R.F.; FRANCO, M.C.; JUNIOR, C.K.F. SANTOS, M.E.L. Técnica modificada para realização da gastro-jejunostomia endoscópica percutânea. Rev. Col. Bras. Cir. 2017; 44(4): 413-415. Disponível em: http://www.scielo.br/scielo.php?script=sci arttext\&pid=S010069912017000400413. Acesso em: 11 de julho de 2019.

TYNG, C.J.; SANTOS, E.F.V.; GUERRA, L.F.A.; BITENCOURT, A.G.V.; 


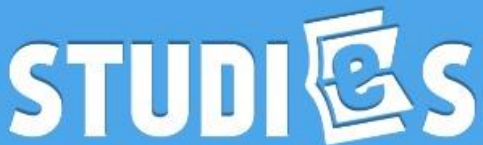

BARBOSA, P.N.V.P.; CHOJNIAK, R. Gastrostomia percutânea guiada por tomografia computadorizada: experiência inicial de um centro oncológico. Radiol Bras. 2017. Disponível em: http://www.scielo.br/pdf/rb/v50n2/pt 01003984-rb-20150219.pdf. Acesso em: 15 de maio de 2019.

ANEXO 1 - ESCALA ANALÓGICA DA FRAGILIDADE CLÍNICO-FUNCIONAL

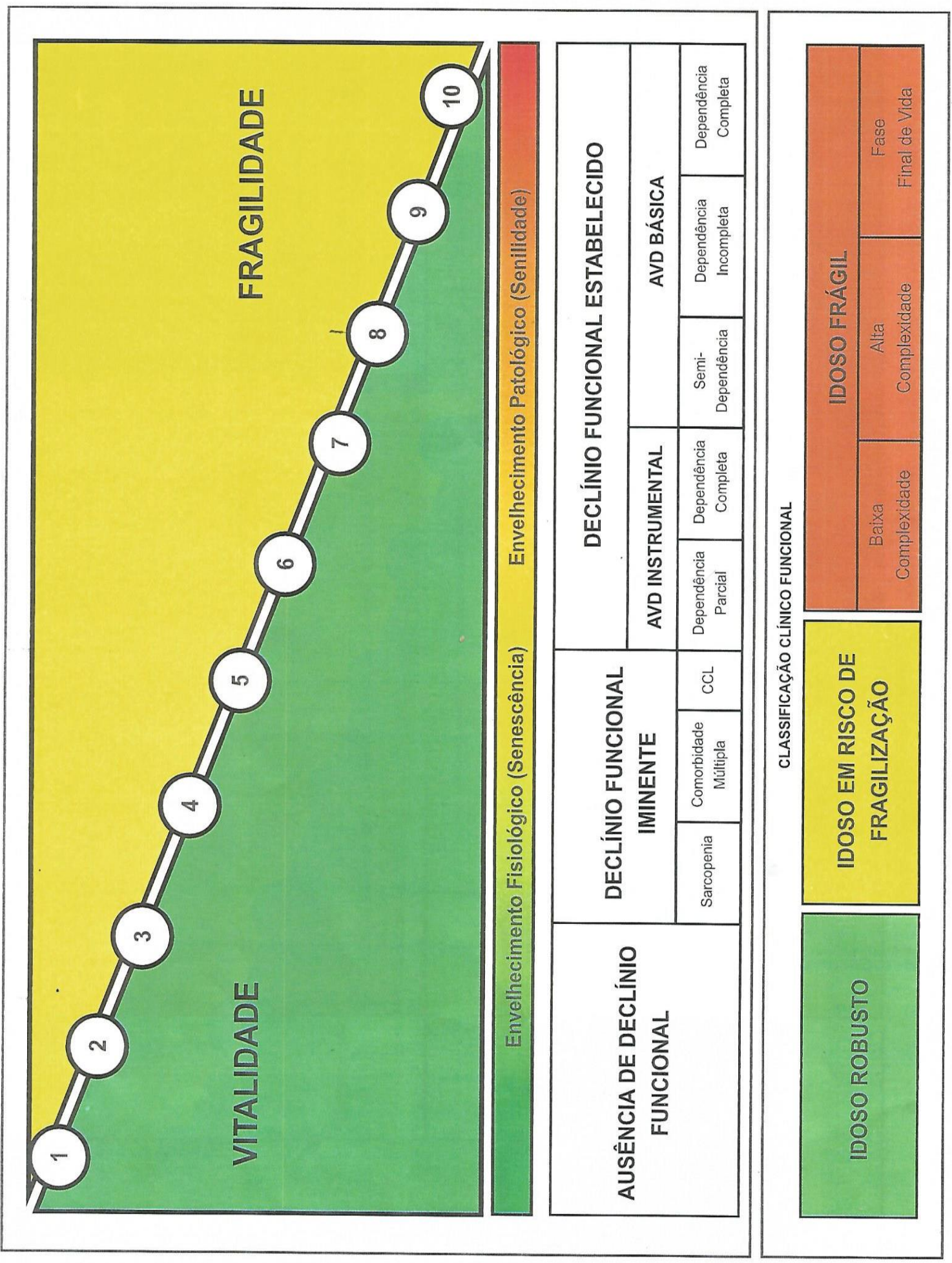




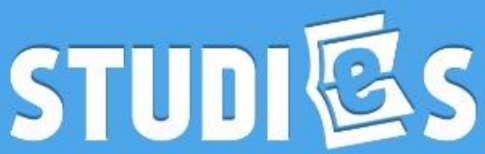 \\ $P \cup B L I C A C O ̈ E S$}

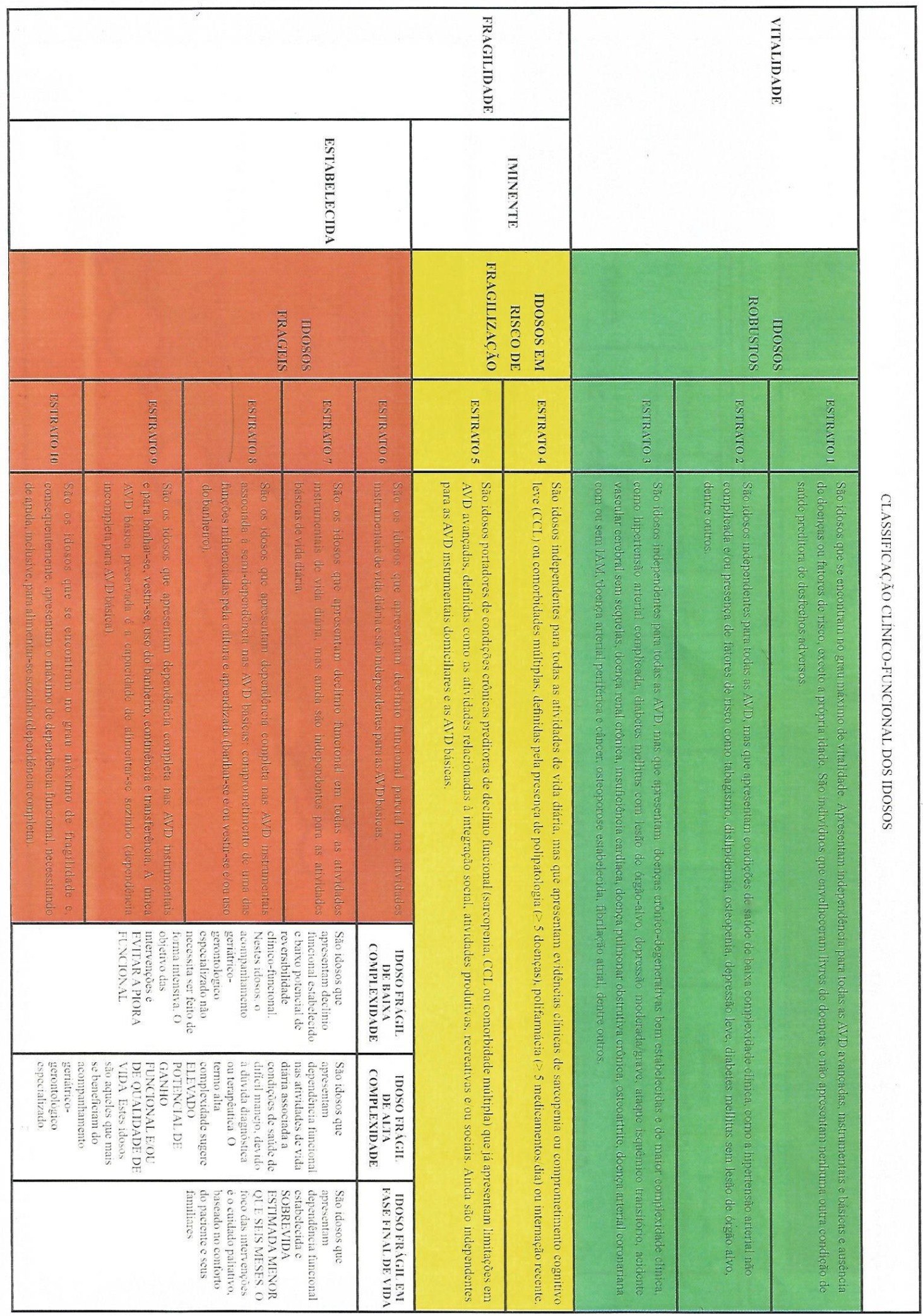

